\title{
Cardiac autophagy is a maladaptive response to hemodynamic stress
}

\author{
Hongxin Zhu, ${ }^{1}$ Paul Tannous, ${ }^{1}$ Janet L. Johnstone, ${ }^{1}$ Yongli Kong, ${ }^{1}$ John M. Shelton, ${ }^{1}$ \\ James A. Richardson, ${ }^{2,3}$ Vien Le, ${ }^{1}$ Beth Levine, ${ }^{1}$ Beverly A. Rothermel, ${ }^{1}$ and Joseph A. Hill1,3,4 \\ 1Department of Internal Medicine, 2Department of Pathology, ${ }^{3}$ Department of Molecular Biology, and \\ ${ }^{4}$ Donald W. Reynolds Cardiovascular Clinical Research Center, University of Texas Southwestern Medical Center, Dallas, Texas, USA.
}

\begin{abstract}
Cardiac hypertrophy is a major predictor of heart failure and a prevalent disorder with high mortality. Little is known, however, regarding mechanisms governing the transition from stable cardiac hypertrophy to decompensated heart failure. Here, we tested the role of autophagy, a conserved pathway mediating bulk degradation of long-lived proteins and cellular organelles that can lead to cell death. To quantify autophagic activity, we engineered a line of "autophagy reporter" mice and confirmed that cardiomyocyte autophagy can be induced by short-term nutrient deprivation in vivo. Pressure overload induced by aortic banding induced heart failure and greatly increased cardiac autophagy. Load-induced autophagic activity peaked at 48 hours and remained significantly elevated for at least 3 weeks. In addition, autophagic activity was not spatially homogeneous but rather was seen at particularly high levels in basal septum. Heterozygous disruption of the gene coding for Beclin 1, a protein required for early autophagosome formation, decreased cardiomyocyte autophagy and diminished pathological remodeling induced by severe pressure stress. Conversely, Beclin 1 overexpression heightened autophagic activity and accentuated pathological remodeling. Taken together, these findings implicate autophagy in the pathogenesis of load-induced heart failure and suggest it may be a target for novel therapeutic intervention.
\end{abstract}

\section{Introduction}

In response to stress from neurohumoral activation, hypertension, or other myocardial injury, the heart initially compensates with an adaptive hypertrophic increase in cardiac mass. Under prolonged stress, the heart undergoes apparently irreversible change resulting in dilation and diminished performance. In epidemiological studies, pathological cardiac hypertrophy is a major predictor of heart failure, the leading hospital discharge diagnosis in the US and a disorder whose mortality remains high at approximately $50 \%$ at 5 years (1). Despite the prominence of hypertrophy as a prelude to heart failure, mechanisms governing the transition from hypertrophy to failure are poorly understood.

Strict regulation of protein turnover is critical in long-lived postmitotic cells such as cardiac myocytes, in which the ability to replace cells is limited although intracellular proteins and organelles recycle continuously. During hypertrophic growth, enhanced protein synthesis leads to an increase in the size of individual myocytes and heightened organization of the sarcomere. Decompensation, on the other hand, is associated with thinning of the ventricular walls through a combination of proteolysis and/or myocyte death with subsequent replacement by fibrotic tissue. Numerous signaling pathways have been causally implicated in stress-induced remodeling of the heart $(2,3)$, and many of these same pathways have also been implicated in cell death.

Nonstandard abbreviations used: Atg8, autophagy-related protein 8; BW, body weight; EGFP, enhanced GFP; \%FS, percent fractional shortening; HW/BW, heart weight normalized to body weight; LAMP-1, lysosomal-associated membrane protein-1; LC3, microtubule-associated protein 1 light chain 3; LVEDD, LV end-diastolic diameter; LVESD, LV end-systolic diameter; $\alpha$-MHC, $\alpha$-myosin heavy chain; mLC3, mouse LC3; rLC3, rat LC3; RT, room temperature; sTAB, severe TAB; TAB, thoracic aortic banding.

Conflict of interest: The authors have declared that no conflict of interest exists. Citation for this article: J. Clin. Invest. 117:1782-1793 (2007). doi:10.1172/JCI27523.
Autophagy is a highly conserved cellular mechanism of protein recycling that can lead to programmed cell death (so-called type II programmed cell death). Autophagy can be induced by starvation, hypoxia, intracellular stress, hormones, or developmental signals (4-6). It is involved in the turnover of mitochondria and peroxisomes and is the major lysosomal pathway for the nonselective delivery of cytoplasmic components during periods of starvation or stress $(5,7)$. In certain contexts, such as nutrient deprivation, autophagic activity is adaptive, as degradation of cytosolic components provides amino acids and substrates for intermediary metabolism. Also, autophagy can eliminate damaged proteins and organelles that might otherwise be toxic or trigger apoptotic death. In other settings, however, dysregulated autophagy may contribute to the pathogenesis of disease (4), including neurodegenerative disorders $(8-10)$, skeletal myopathy, cancer $(11,12)$, and infectious diseases (13). Nothing is known about the possible role of autophagy in the most common form of heart failure, that induced by hemodynamic stress.

Here, we report for the first time to our knowledge that pressureoverload stress - a major risk factor for cardiac hypertrophy and failure - triggers cardiomyocyte autophagy. We provide evidence that beclin 1 (Becn1), the mammalian homolog of a proautophagic gene in yeast, is a critical factor required for cardiac autophagy. Finally, by modulating cellular expression of beclin 1 in both positive and negative directions, we titrated the autophagic response to pressure overload, providing direct evidence that load-triggered cardiac autophagy is a maladaptive response that contributes to heart failure progression.

\section{Results}

Myocyte autophagy triggered by nutrient deprivation. During autophagy, LC3 (microtubule-associated protein 1 light chain 3), an $18-\mathrm{kDa}$ homolog of autophagy-related protein 8 (Atg8) in yeast, is 


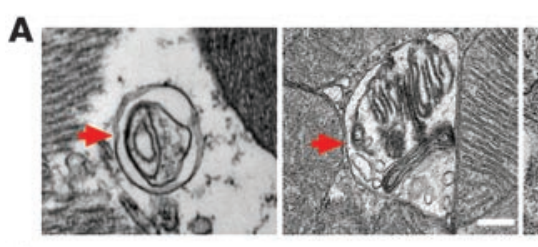

C

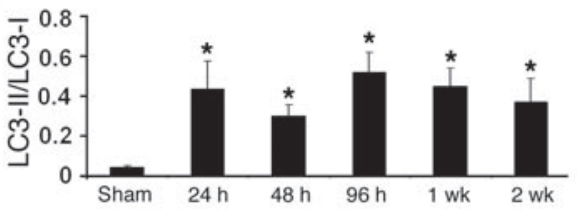

E

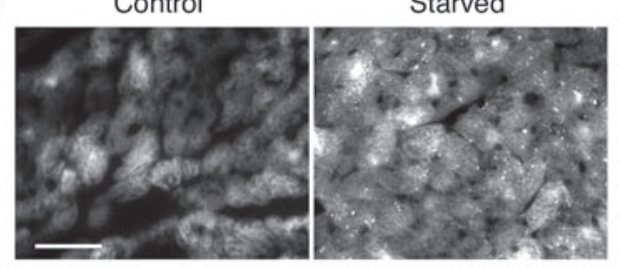

G

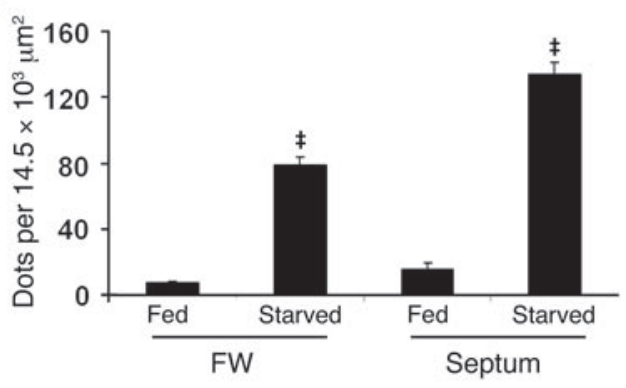

B

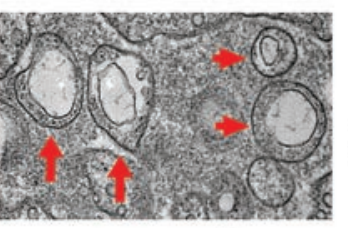

D

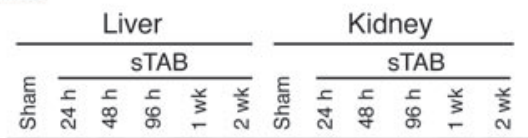

LC3-I

LC3-II

F

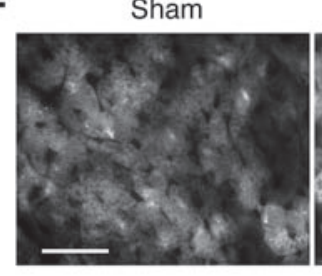

STAB

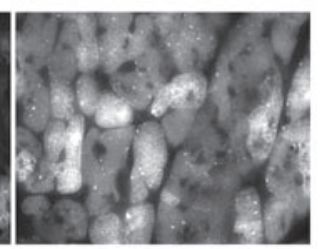

H

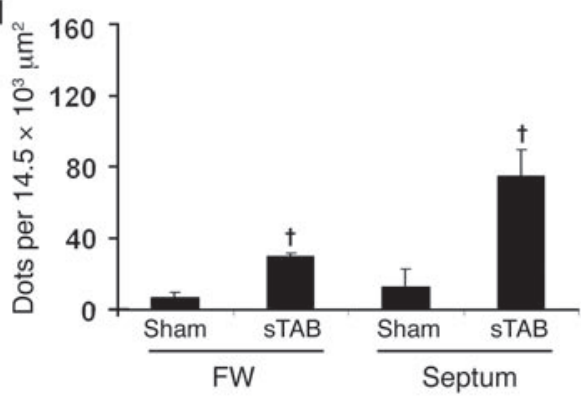

Figure 1

Cardiomyocyte autophagy triggered by short-term nutrient deprivation or pressure-overload hemodynamic stress. (A) Representative electron micrographs from the septa of 48-hour STAB LVs (WT C57BL/6 mice) demonstrate the presence of double-membrane autophagosomes (arrows) and autolysosomes containing cellular material. These features are more prominent in STAB ventricles compared with those of sham-operated controls. Scale bar: $120 \mathrm{~nm}$. (B) Representative immunoblot for LC3 showing an increase in LC3-II abundance following sTAB as early as 24 hours after operations and persisting for at least 2 weeks. (C) Quantification of LC3-II/LC3-I levels demonstrates significant autophagic activity induced by pressure overload. ${ }^{\star} P<0.05$ versus Sham. (D) Representative immunoblots for LC3 in liver and kidney demonstrating that LC3-II abundance does not change in these tissues following STAB. (E) Under baseline conditions, GFP-LC3 Tg fusion protein is diffusely distributed throughout the cardiomyocyte cytoplasm in $\alpha$-MHC-GFP-LC3 mice. Following short-term (48 hours) starvation, GFP-LC3 aggregates as autophagosome-localized GFP dots. Representative images from basal septum are shown. Scale bar: $35 \mu \mathrm{m}$. (F) Following sham operation, GFP-LC3 Tg fusion protein is diffusely distributed throughout the cardiomyocyte cytoplasm in $\alpha$-MHC-GFP-LC3 mice. Following imposition of pressure stress by STAB (48 hours), GFP-LC3 aggregates as autophagosome-localized GFP dots. Representative images from basal septum are shown. Scale bar: $35 \mu \mathrm{m}$. (G) Quantification of GFP aggregates per microscopic field $\left(14,479 \mu \mathrm{m}^{2}\right)$ demonstrates significant autophagic activity induced by starvation. For each group, at least 4 mice were studied. $\neq P<0.01$ versus fed. (H) Quantification of GFP aggregates per microscopic field $\left(14,479 \mu \mathrm{m}^{2}\right)$ demonstrates significant autophagic activity induced by pressure overload. For each group, at least 4 mice were studied. ${ }^{\dagger} P<0.01$ versus sham. FW, free wall of LVs.

processed and lipid conjugated (14). The resulting $16-\mathrm{kDa}$ active isoform migrates from the cytoplasm to isolation membranes and autophagosomes. Recently, intracellular processing of rat LC3 (rLC3) has emerged as a reliable marker of autophagic activity (15). We confirmed our ability to detect cardiomyocyte autophagic activity in response to the established autophagy trigger, shortterm nutrient deprivation (details provided in supplemental material; available online with this article; doi:10.1172/JCI27523DS1).

Load-induced cardiac autophagy. Afterload stress, such as that imposed by chronic hypertension, is a major cause of heart failure (16). To assess the potential contribution of autophagy to the fail- ure response of pressure-stressed ventricle, we studied mice with pressure-overload heart failure. Severe afterload stress was induced by surgical constriction of the proximal aorta (severe thoracic aortic banding [sTAB]), a model of load-induced heart failure (17). Perioperative (24 hours) and 1-week mortalities ( $<10 \% ; P=\mathrm{NS}$ ) were similar to those following standard TAB (18), but morphological and functional indicators demonstrated systolic dysfunction and clinical heart failure (17). Electron micrographic analysis of ventricular tissue was used to identify double membrane-bound autophagic vesicles. Electron micrographs from the septa of 48-hour STAB hearts revealed extensive vacuolization (Figure 1A), 
A

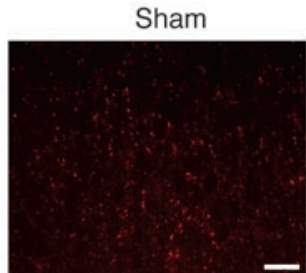

$96 \mathrm{~h}$

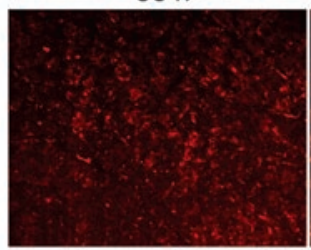

B

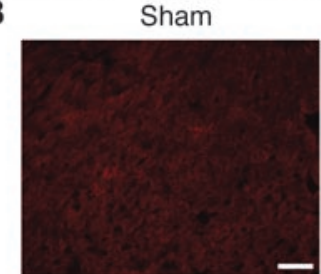

$96 \mathrm{~h}$

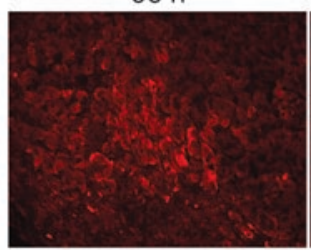

C

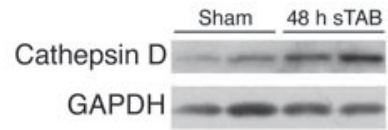

$24 \mathrm{~h}$

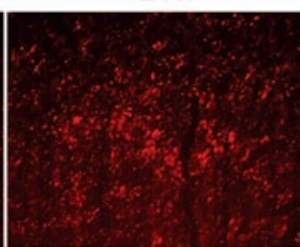

1 wk

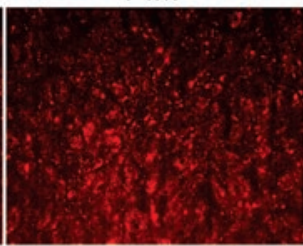

$24 \mathrm{~h}$

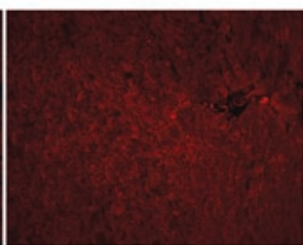

1 wk
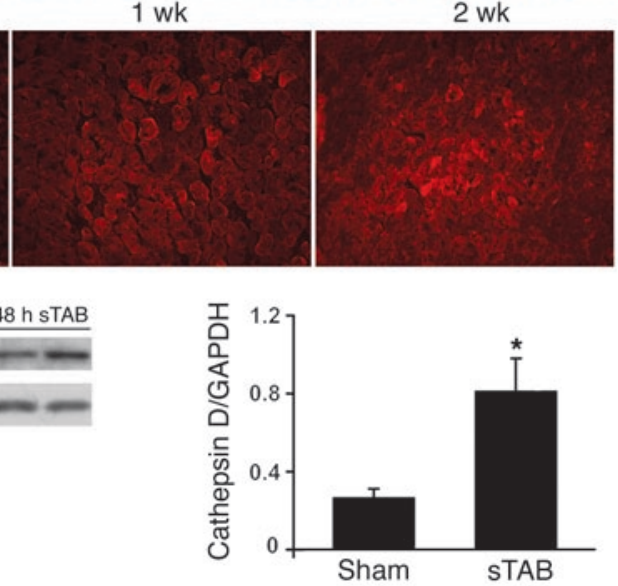

Figure 2

Increased abundance of lysosomal markers in STAB ventricle. LAMP-1 (A) and cathepsin D (B), detected by immunohistochemistry, are increased in STAB ventricle at multiple time points, indicative of increased lysosomal activity in pressure-stressed LVs. Scale bars: $40 \mu \mathrm{m}$. (C) Representative immunoblot of ventricular lysates from sham-operated and STAB LVs probed for cathepsin D. Mean data from 3 independent experiments. ${ }^{\star} P<0.05$.

Under basal conditions, GFP fluorescence was diffusely distributed throughout the cardiomyocyte cytoplasm (Figure 1E). Fasting the autophagy-reporter mice (48 hours), however, resulted in a dramatic increase in the abundance of GFP-positive vesicles (Figure 1, E and G), verifying that the $\mathrm{Tg}$ responded appropriately in vivo. Significant increases in autophagic activity were detected throughout the starved LVs. Interestingly, however, in response to starvation, a preponderance of autophagic activity was consistently detected in the basal region of the interventricular septum. Consistent with other studies $(15,19)$, we detected no evidence of increased autophagic activity in 2 independent lines of LC3-overexpressing mice compared with WT mice, consistent with the fact that forced expression alone of GFP-LC3 is insufficient to trigger autophagy (see below).

To determine whether pressure overload triggers cardiomyocyte autophagy, $\alpha$-MHC-GFP-LC3 mice were subjected to sTAB. In sham-operated LVs, cardiomyocyte GFP-LC3 abundance and distribution were each similar to that in unoperated controls; GFP fluorescence was distributed diffusely throughout the cytoplasm with few punctate aggregates (Figure 1F). Twenty-four hours after banding, however, punctate GFP-LC3 was significantly increased, indicating increased autophagic activity. Just as in the setting of starvation-triggered autophagy, we observed a striking preponderance of GFP aggregation in the basal septum of the LV (Figure $1 \mathrm{H})$.

As noted earlier, prior studies have demonstrated that GFP-LC3 overexpression, both in vitro and in vivo, does

similar to that observed in starved LVs. These features were more prominent in STAB hearts compared with those of sham-operated controls. Evidence for increased autophagic activity was also obtained by Western blot, which revealed increases in LC3-II levels occurring as early as 24 hours after sTAB, with levels remaining elevated for at least 2 weeks (Figure 1, B and C). LC3 processing was not detected in liver or kidney (Figure 1D), indicating that the pressure overload-induced autophagic response is specific to heart and not reflective of malaise, neurohumoral activation, or other global responses to stress.

Ultrastructural analysis by electron microscopy is labor intensive and expensive, and the prevalence of autophagic features is difficult to quantify. Further, biochemical analyses on ventricular lysates do not distinguish events occurring in cardiomyocytes from those occurring in nonmyocyte elements of the LV. Thus, to study and quantify cardiomyocyte autophagy, a rapid, reliable bioassay is required. To address this need, we engineered a line of "autophagy reporter" mice that express GFP-tagged mouse LC3 (mLC3) under the control of a cardiomyocyte-specific, $\alpha$-myosin heavy chain promoter ( $\alpha$-MHC-GFP-LC3). not alter basal or starvation-induced autophagy $(15,19)$. To determine whether overexpression of GFP-LC3 alters the remodeling response to pressure-overload stress, we performed echocardiographic and necropsy studies on $\alpha$-MHC-GFP-LC3 mice subjected to STAB. In these experiments, we detected no differences in the hypertrophic growth response of the $\mathrm{Tg}$ animals relative to that of WT mice (Supplemental Table 1). Further, functional indices of cardiac performance were similar across the genotypes. We conclude that cardiomyocyte-restricted expression of GFP-LC3 does not alter the response to pressure stress and affords a valid readout to track and quantify autophagic activity in the heart.

The increase in autophagosome-localized GFP-LC3 in stressed cardiac myocytes indicates autophagosome accumulation (14, $20)$. To determine whether this was due to an increase in autophagosome formation or was a secondary consequence of lysosome dysfunction preventing autophagosome clearance, we performed immunohistochemistry for 2 lysosomal markers, lysosomal-associated membrane protein-1 (LAMP-1) and cathepsin D. In each case, we found that lysosome abundance was increased in failing heart relative to control for at least 2 weeks (Figure 2, A and B), sug- 


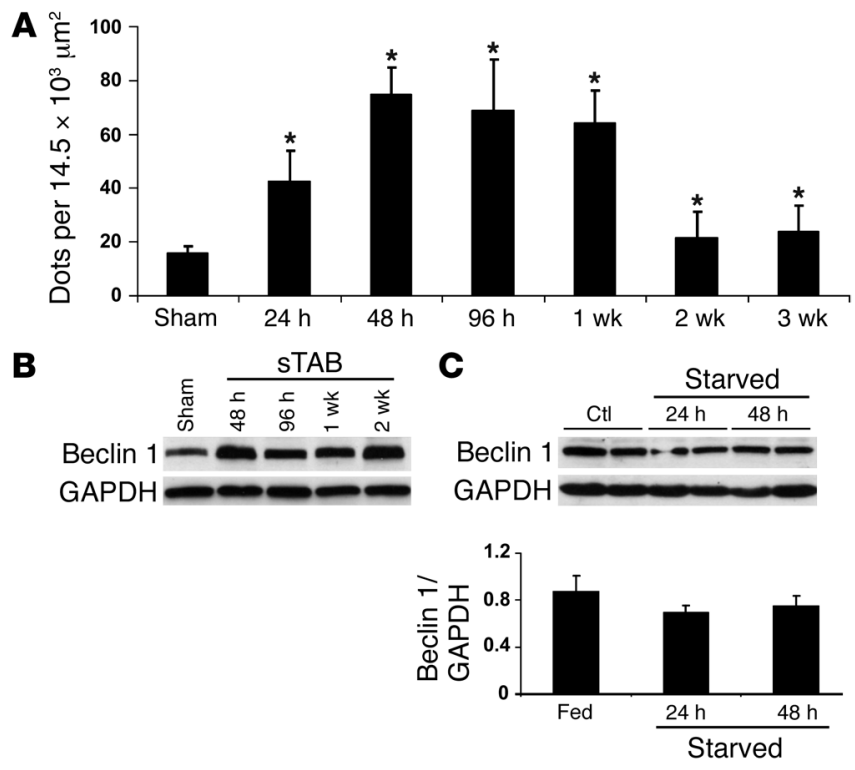

gesting the diminished lysosomal function did not contribute to autophagosome accumulation in stressed cardiomyocytes. Similar results were obtained by immunoblot (Figure $2 \mathrm{C}$ ). Together, these data are consistent with an increase in flux through autophagic clearance pathways.

Autophagic activity, detected either as LC3 processing on immunoblot or using GFP-LC3 Tgs, reached a peak at 48 hours following STAB and remained significantly elevated for at least 3 weeks (Figure 1B and Figure 3A). Considering the obligatory role of beclin 1, the mammalian homolog of the proautophagic gene Atg6 in yeast, in the recruitment of Atg12-Atg5 conjugates to preautophagosomal membranes (21), we measured Beclin 1 protein levels in hearts with increased autophagic activity. Beclin 1 abundance was increased in pressure-stressed LVs. (Figure 3B). Interestingly, although starvation induced an autophagic response comparable in degree to STAB (see below), Beclin 1 protein levels were not altered in the starved hearts (Figure 3C).

Recent studies have uncovered significant interactions between autophagic and apoptotic signaling pathways $(19,22)$. Further, apoptosis has been implicated in the overall process of myocardial remodeling and the transition from cardiac

\section{Figure 4}

Pressure overload induces apoptosis in proximal aorta but not in LVs. (A) Rare TUNEL-positive figures (pink circles) are detected in ventricular myocardium from sham-operated or STAB hearts (basal septum) whereas a significantly increased signal is detected in aorta. Scale bar: $10 \mu \mathrm{m}$. (B) Microscopic fields (10-15 per section), each containing approximately 800 myocytes, were evaluated by TUNEL staining ( $n=30-45$ fields from each of 3 mice at each time point) in C57BL/6 mice. $P=\mathrm{NS}$ for each time point compared with sham. (C) DNA laddering (arrows) indicative of apoptosis in proximal aortic tissue (Ao), but not LVs, in animals subjected to sTAB.

\section{Figure 3}

Time course of autophagic activity in STAB ventricle and changes in Beclin 1 protein levels. (A) Autophagic activity induced in the basal septum in response to STAB was quantified by counting GFP-LC3 dots per microscopic field $\left(14,479 \mu \mathrm{m}^{2}\right)$. Representative immunoblot for Beclin 1 showed an increase in Beclin 1 abundance following STAB (B) but not in response to short-term nutrient deprivation (C). ${ }^{*} P<0.05$ versus sham. Ctl, control.

hypertrophy to failure (23-25) although its relevance in acute and chronic heart disease is the subject of debate (26). To address the potential contribution of apoptosis to the remodeling of pressure-stressed ventricle, we studied sTAB ventricle (48 hours) using TUNEL staining and nucleosomal DNA laddering. Both approaches revealed evidence for apoptosis in the aorta proximal to the surgical constriction but not in the myocardium (Figure 4). Together, these findings suggest that apoptotic cell death, clearly detected in the proximal aorta, is not a major mechanism of ventricular remodeling at the disease stage (48 hours after STAB) that we have studied here.

Beclin 1 disruption decreases cardiomyocyte autophagy in vivo. Autophagy is a mechanism required for normal development and, depending on the context, an adaptive response to stress; in other settings, autophagy is maladaptive, contributing to disease pathogenesis and cell death $(19,27,28)$. It is not known whether cardiac autophagy triggered by hemodynamic stress is adaptive or maladaptive. Homozygous disruption of beclin 1 is embryonic lethal, but mice subjected to heterozygous disruption of beclin 1 (beclin $1^{+-}$) are viable, manifesting diminished autophagy in multiple tissues (12). We hypothesized that beclin $1^{+/-}$mice would also manifest a diminished autophagic response in the heart. To test this, we crossed the autophagy-reporter line with beclin $1^{+/-}$mice and quantified autophagic activity induced by short-term nutrient deprivation. In these experiments, we found that autophagic activity triggered

A

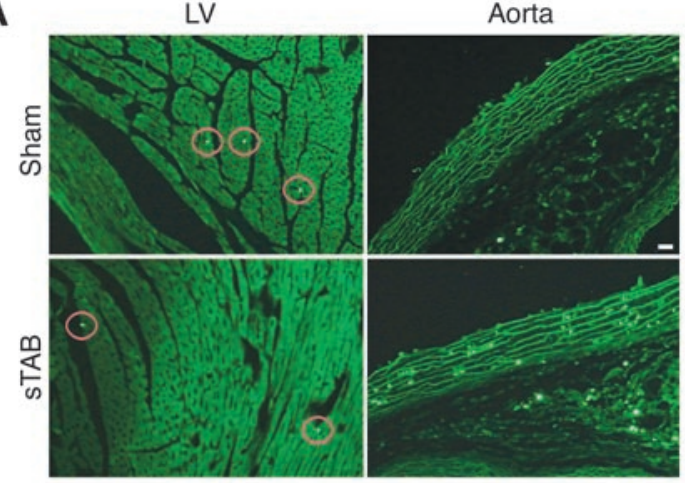

B

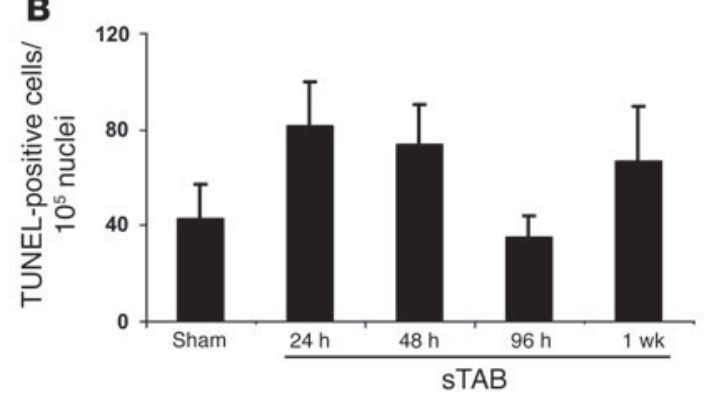

C

Sham STAB

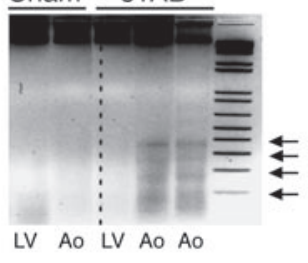


A

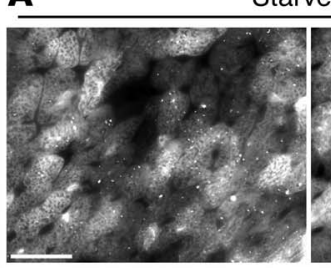

$\alpha-\mathrm{MHC}-\mathrm{LC} 3$

B

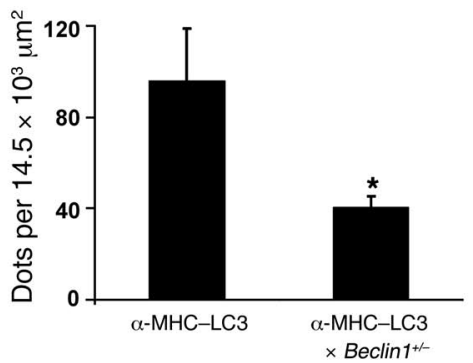

C

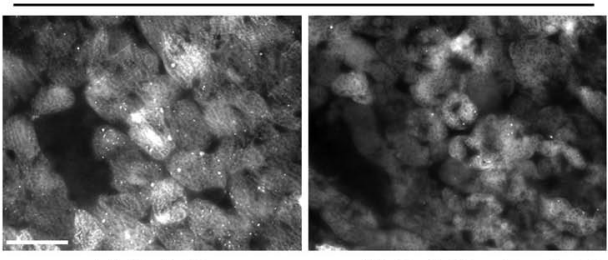

$\alpha-\mathrm{MHC}-\mathrm{LC} 3$

$\alpha-\mathrm{MHC}-\mathrm{LC} 3 \times$ Beclin1 $^{+1}$

D

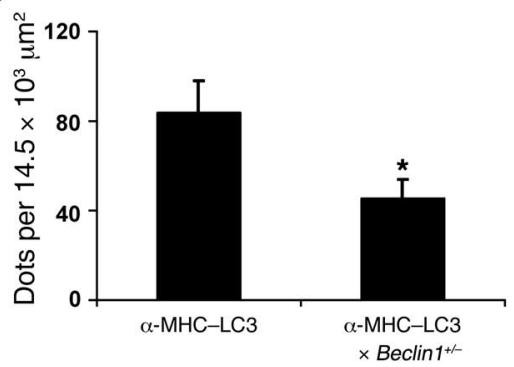

Figure 5

Heterozygous disruption of beclin 1 decreases cardiomyocyte autophagy. (A) Autophagy induced by starvation (48 hours), manifested as punctate GFP-LC3 dots, is significantly diminished in beclin $1^{+/-}$hearts. All images were taken from basal septum. Scale bar: $35 \mu \mathrm{m}$. (B) Quantification of GFP aggregates per microscopic field $\left(14,479 \mu \mathrm{m}^{2}\right)$ in basal septum demonstrates significantly less autophagic activity in beclin $1^{+/-}$hearts in response to starvation compared with WT. $n=3-5$ microscopic fields in each of 3 mice. (C) Induction of autophagy by pressure overload (sTAB, 48 hours) is significantly diminished in beclin $1^{+/-}$hearts compared with WT. All images are taken from basal septum. Scale bar: $35 \mu \mathrm{m}$. (D) Quantification of GFP aggregates per microscopic field $\left(14,479 \mu \mathrm{m}^{2}\right.$, basal septum) demonstrated significantly less autophagic activity in beclin $1^{+/-}$ hearts exposed to hemodynamic stress. $n=3-5$ microscopic fields in each of 3 mice. ${ }^{*} P<0.05$ versus $\alpha-$ MHC-GFP-LC3 mice.

by short-term starvation was significantly diminished $(P<0.05)$ by heterozygous inactivation of the beclin 1 gene (Figure 5, A and B).

To determine whether beclin 1 inactivation alters the autophagic response to pressure stress, we evaluated cardiomyocyte autophagy in hearts subjected to STAB (48 hours). Responding similarly to cardiomyocytes that experienced starvation, cardiomyocytes in the beclin $1^{+/}$- background manifested significantly fewer GFP-LC3 punctate "dots" as compared with beclin 1 WT controls (Figure 5, C and D). Quantification of autophagic activity at 1 week after sTAB revealed similar decreases in beclin $1^{+/-}$mice (GFP-LC3 dots per $14.5 \times 10^{3} \mathrm{~mm}^{2}$ : beclin $1^{+/}, 30 \pm 5, n=3$; WT, $66 \pm 12, n=5 ; P<0.01$ ). As with WT controls, pressure overload did not trigger autophagy in liver, kidney, or brain of beclin $1^{+/-}$mice (Supplemental Figure 4). Lysosomal abundance was decreased in beclin $1^{+/-}$hearts, consistent with diminished flux through autophagy clearance pathways (Supplemental Figure 5). TUNEL staining and nucleosomal DNA laddering did not reveal evidence of significant activation of apoptotic pathways in beclin $1^{+/-}$LVs (data not shown); these findings were very similar to our results in WT heart subjected to sTAB. Together, these results lend support to our finding of loadinduced cardiac autophagy and point to Beclin 1 as a required element in the cascade of events leading to autophagy in the heart.

Animals subjected to STAB develop heart failure, manifested clinically as lethargy, diminished mobility, and sudden death (17). To determine whether STAB-induced increases in autophagy derive from inability to consume and absorb nutrients, we monitored body weight (BW) for the first 48 hours after STAB, prior to the development of edema. Whereas animals deprived of food lost substantial weight during this period, sTAB animals did not (Supplemental Figure 6). Thus, autophagic activation after sTAB does not stem from caloric deprivation.
Because our results showed that cardiomyocyte autophagy was diminished in beclin $1^{+/-}$hearts subjected to pressure stress (Figure 5, C and D) and that load stress leads to increases in Beclin 1 abundance (Figure 3B), we measured Beclin 1 levels in beclin $1^{+/-}$hearts following sTAB surgery. First, Beclin 1 levels increased in both WT and beclin $1^{+/}$hearts following imposition of pressure overload, as expected (Figure $3 \mathrm{~B}$ and Figure $6 \mathrm{~A})$. However, Beclin 1 protein was consistently less abundant in beclin $1^{+/}$hearts following sTAB relative to WT hearts (Figure $6 \mathrm{~B})$, consistent with the haploinsufficient genotype. We concluded that the beclin 1 haploinsufficiency model is validated in studies designed to titrate the autophagic response to imposed hemodynamic load.

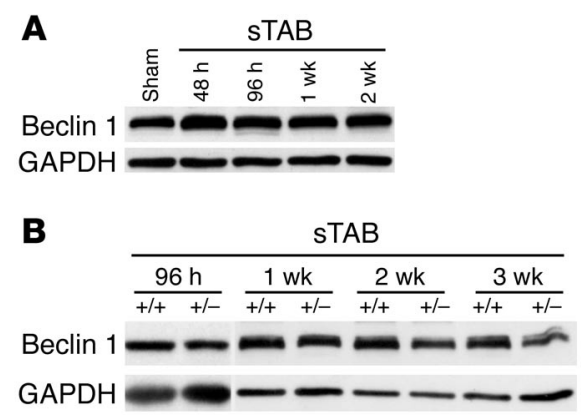

Figure 6

Beclin 1 levels increase with pressure-overload stress but remain lower in beclin $1^{+/-}$hearts relative to WT. (A) Representative immunoblot demonstrating a time course of Beclin 1 levels following sTAB surgery in beclin $1^{+-}$hearts. (B) Direct comparisons of Beclin 1 protein levels in WT and beclin $1^{+/-}$hearts following STAB surgery. 

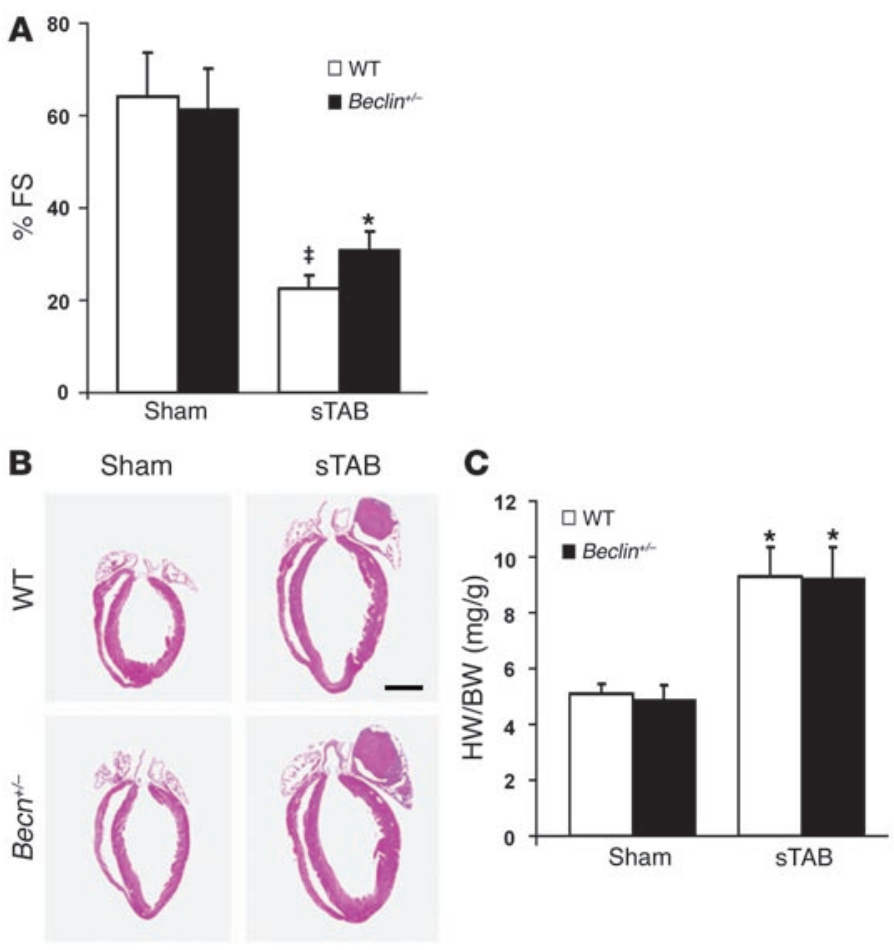

Beclin 1 disruption limits pathological remodeling in pressure-stressed $L V s$. To determine whether load-induced autophagy contributes to pathological ventricular remodeling, we evaluated LV size and systolic function in beclin $1^{+/-}$hearts subjected to pressure-overload stress. To maximize the sensitivity of the readout, we performed echocardiography under conditions of light conscious sedation, and we evaluated hearts at a late time point, when the disease phenotype was well established. In addition, we performed invasive hemodynamic measurements in anesthetized mice. At 3 weeks following STAB, systolic performance in WT animals was significantly decreased, consistent with our previous findings (17). In beclin $1^{+/}$mice, however, pressure overload-induced declines in systolic function were attenuated (Figure 7A and Table 1$)$. On average, WT mice manifested a $68 \%( \pm 2 \%)$ decline in percent fractional shortening (\%FS) at 3 weeks following sTAB. In contrast, declines in systolic performance in beclin $1^{+-}$ mice were blunted $(55 \% \pm 7 \%)$, a difference from WT mice that was modest but statistically highly significant $(P<0.01)$. Ventricular dilation at end-diastole was similar in WT and beclin $1^{+/-}$ mice (LV end-diastolic diameter [LVEDD] $/ \mathrm{mm}: 4.87 \pm 0.5$, WT, $n=6$, versus $4.82 \pm 0.6$ beclin $\left.1^{+/-}, n=6 ; P=\mathrm{NS}\right)$. However, a trend toward preservation of end-systolic dimensions was detected in beclin $1^{+/}$mice following STAB (LV end-systolic diameter [LVESD] $/ \mathrm{mm}: 3.77 \pm 0.4$, WT, $n=6$, versus $3.33 \pm 0.4$ beclin $1^{+/-}$, $n=6 ; P=0.07)$. Heart mass on necropsy was increased similarly in the WT and beclin $1^{+/}$backgrounds, suggesting that compensatory ventricular growth was not altered at the 3-week time point (Figure 7, B and C). Similar results were obtained when beclin $1^{+/-}$ mice were subjected to the moderate pressure stress of standard $\mathrm{TAB}$; increases in heart mass on necropsy were similar relative to WT (heart weight normalized to BW [HW/BW]: $4.7 \pm 0.2$ beclin $1^{+/-}$sham, $n=4 ; 7.7 \pm 1.2$ beclin $1^{+/-} \mathrm{TAB}, n=4 ; 5.1 \pm 0.1 \mathrm{WT}$ sham, $n=4 ; 7.1 \pm 0.8 \mathrm{WT} \mathrm{TAB}, n=4 ; P=\mathrm{NS}$ ). Thus, downregula-

\section{Figure 7}

Pathological remodeling in pressure-stressed ventricle is diminished when autophagy is inhibited by beclin 1 haploinsufficiency. (A) Pressure overload-induced declines in systolic function, measured as \%FS, are significantly decreased in beclin $1^{+/-}$mice. Systolic performance was measured at 3 weeks after banding. $n=4$ WT sham; $n=4 \pm$ sham; $n=6$ WT sTAB; $n=6 \pm$ sTAB. (B) Four-chamber sections of hearts treated as listed and harvested at 3 weeks. Scale bar: $2 \mathrm{~mm}$. (C) HW/BW is increased similarly in banded beclin $1^{+/-}$mice compared with WT controls. $n=6$ WT sham; $n=10 \pm$ sham; $n=7$ WT sTAB; $n=8 \pm$ sTAB. $\ddagger P<0.05$ versus \pm STAB; ${ }^{*} P<0.05$ versus \pm sham.

tion of autophagy by beclin 1 inactivation diminished pathological declines in systolic performance triggered by pressure overload while compensatory hypertrophic growth remained similar. This, then, is consistent with the hypothesis that load-induced cardiac autophagy contributes to pathological ventricular remodeling triggered by hemodynamic stress.

Beclin 1 promotes autophagy and pathological ventricular remodeling. In human breast carcinoma cells, Beclin 1 overexpression promotes autophagy (29). To test the hypothesis that loadinduced cardiomyocyte autophagy is maladaptive, we generated $\mathrm{Tg}$ mice that overexpress Beclin 1 driven by the cardiomyocyte-specific $\alpha$-MHC promoter. Beclin 1 overexpression elicited modest increases in autophagic activity under resting conditions (Figure 8, A and B). Also, starvation-induced increases in autophagy were significantly amplified in beclin $1 \mathrm{Tg}$ hearts (Figure 8 , A and B). Thus, Beclin 1 overexpression is sufficient to augment stress-induced cardiomyocyte autophagy.

To study the effects of increased autophagy on cardiac remodeling, we first determined whether the modest increases in autophagy present under basal conditions in beclin 1 Tgs was sufficient to induce ventricular remodeling. Ventricular size and performance were studied in mice up to 10 months of age. In beclin 1 Tgs, systolic function measured as \%FS by echocardiography was normal (WT: $62 \pm 6 \%, n=6$; Tg: $63 \pm 8 \%, n=6$; $P=\mathrm{NS}$ ), and ventricular size was unchanged (data not shown). Also, the fetal gene program was not activated in beclin $1 \mathrm{Tg}$ hearts (Supplemental Figure 7). Together, these data are consistent with a lack of significant effect of Beclin 1 overexpression on ventricular remodeling under resting conditions.

To study the effects of increased autophagy on stress-triggered remodeling, we exposed beclin $1 \mathrm{Tg}$ mice to pressure stress. First, Beclin $1 \mathrm{Tg}$ mice $(n=7)$ subjected to severe pressure stress (sTAB) manifested profoundly increased mortality (14\% survival at 1 week, $n=7$ ) relative to WT mice ( $100 \%$ survival at 1 week, $n=6)$, consistent with augmented pathological remodeling in the beclin 1 Tgs. Next, we subjected animals to standard TAB, a procedure that induces stable ventricular hypertrophy without failure (18). First, we evaluated lysosomal function by measuring the lysosomal markers LAMP-1 and cathepsin D. In each case, we found that lysosome abundance was unchanged in sham-operated beclin $1 \mathrm{Tgs}$ (Figure 9, A and B). Following TAB, however, lysosome abundance was increased in WT hearts and to a greater extent in beclin $1 \mathrm{Tg}$ hearts. Similar results were obtained by immunoblot (Figure 9C). Also, increased levels of apoptotic cell death were not detected in beclin $1 \mathrm{Tg}$ LVs under both sham-operated and TAB conditions (data not shown). Similar to our findings with STAB ventricles, these data are consistent with an increase in flux through autopha- 


\section{Table 1}

Echocardiographic, morphometric, and physiological parameters 3 weeks post sTAB surgery

\begin{tabular}{|c|c|c|c|c|}
\hline & \multicolumn{2}{|c|}{ Sham } & \multicolumn{2}{|c|}{ sTAB } \\
\hline & WT & Beclin 1+- & WT & Beclin 1+- \\
\hline \multicolumn{5}{|c|}{ Echocardiographic data } \\
\hline $\mathrm{HR}$ (bpm) & $615 \pm 30$ & $585 \pm 30$ & $540 \pm 95$ & $564 \pm 91$ \\
\hline IVSD (mm) & $0.93 \pm 0.12$ & $0.91 \pm 0.27$ & $1.13 \pm 0.37$ & $1.13 \pm 0.15$ \\
\hline LVPW (mm) & $1.22 \pm 0.18$ & $1.28 \pm 0.11$ & $1.38 \pm 0.21$ & $1.41 \pm 0.29$ \\
\hline LVEDD (mm) & $3.23 \pm 0.50$ & $3.46 \pm 0.33$ & $4.87 \pm 0.51^{A}$ & $4.82 \pm 0.61^{\mathrm{A}}$ \\
\hline LVESD (mm) & $1.27 \pm 0.47$ & $1.22 \pm 0.12$ & $3.77 \pm 0.40^{\mathrm{A}}$ & $3.33 \pm 0.44^{A}$ \\
\hline$\% \mathrm{FS}$ & $64.28 \pm 9.26$ & $61.40 \pm 8.85$ & $22.48 \pm 2.82^{\mathrm{A}}$ & $30.77 \pm 4.26^{\mathrm{B}}$ \\
\hline$n$ (animals) & 4 & 4 & 6 & 6 \\
\hline \multicolumn{5}{|c|}{ Morphometric and physiological data } \\
\hline BW $(g)$ & $25.0 \pm 0.68$ & $24.6 \pm 0.36$ & $24.3 \pm 1.1$ & $24.1 \pm 1.6$ \\
\hline HW/BW & $5.13 \pm 0.31$ & $5.03 \pm 0.46$ & $9.50 \pm 1.07^{A}$ & $9.35 \pm 1.49 \mathrm{~A}$ \\
\hline LW (mg) & $149.7 \pm 9.8$ & $150.8 \pm 12.4$ & $409.4 \pm 83.4^{A}$ & $360 \pm 78^{A}$ \\
\hline $\mathrm{SBP}(\mathrm{mmHg})$ & $135.6 \pm 7.8$ & $135.3 \pm 9.2$ & $\mathrm{~N} / \mathrm{A}$ & $\mathrm{N} / \mathrm{A}$ \\
\hline$n$ (animals) & 6 & 10 & 7 & 8 \\
\hline \multicolumn{5}{|c|}{ Invasive hemodynamics } \\
\hline HR (bpm) & $493 \pm 10$ & $497 \pm 16$ & $446 \pm 33$ & $488 \pm 19$ \\
\hline$d P / d t_{\max }(\mathrm{mmHg} / \mathrm{s})$ & $6,116.5 \pm 485$ & $5,013 \pm 670$ & $2,554 \pm 622^{A}$ & $3,759 \pm 781^{B}$ \\
\hline$d P / d t_{\min }(\mathrm{mmHg} / \mathrm{s})$ & $-6,010.8 \pm 381.6$ & $-5,081.7 \pm 335$ & $-2,157 \pm 720^{A}$ & $-3,301 \pm 858^{B}$ \\
\hline $\operatorname{LVSP}(\mathrm{mmHg})$ & $98.0 \pm 8.1$ & $89.6 \pm 6.3$ & $68 \pm 17.1^{A}$ & $75.8 \pm 13.1^{\mathrm{A}}$ \\
\hline$n$ (animals) & 6 & 4 & 5 & 5 \\
\hline
\end{tabular}

$\mathrm{HR}$, heart rate; IVSD, interventricular septum diameter; LVPW, LV posterior wall thickness; LW, lung weight; SBP, systolic blood pressure by tail cuff; N/A, not available; LVSP, LV systolic pressure by invasive hemodynamic measurement. ${ }^{A} P<0.05$ versus $W T$ sham; ${ }^{B} P<0.05$ versus WT STAB.

gic pathways - i.e., increased autophagy - in TAB LVs and suggest that similar mechanisms of autophagic activation pertain in both moderate (TAB) and severe (sTAB) load-induced cardiac injury.

At 2 weeks after TAB, survival was similar between beclin $1 \mathrm{Tg}$ mice and WT littermates. However, we detected evidence of pathological cell growth, as normalized heart mass of banded beclin $1 \mathrm{Tg}$ mice $(8.9 \pm 1.7, n=6)$ was significantly higher $(P<0.05)$ than that of banded WT controls $(6.3 \pm 0.8, n=8)$ (Figure 10, A and E; Table 2 ). Trichrome staining for reactive fibrosis revealed significant increases in banded beclin $1 \mathrm{Tg}$ heart compared with that of banded WT littermates, consistent with augmentation of pathological ventricular remodeling (Figure 10B). Quantification of GFP aggregates demonstrated significant upregulation of autophagic activity in beclin $1 \mathrm{Tg}$ hearts crossed into the autophagy reporter line and exposed to standard TAB (Figure 10C), suggesting that moderate pressure stress is capable of triggering an autophagic response similar to that caused by severe pressure stress, albeit at lower levels. To test for the presence of myocyte hypertrophy, we quantified cell cross-sectional area in sections of LV tissue (Figure 10D). As expected, cell cross-sectional area in WT mice was significantly increased by TAB. In beclin $1 \mathrm{Tg}$ hearts, TAB-induced increases in myocyte size were significantly greater than in WT hearts. Interestingly, activation of the fetal gene program was comparable in WT and beclin $1 \mathrm{Tg}$ hearts subjected to TAB (Supplemental Figure 7).

To test further for augmented pathological remodeling in the Beclin 1 Tgs, we evaluated ventricular size and systolic performance by echocardiography in animals exposed to TAB. In WT hearts, systolic function in animals subjected to TAB was normal, consistent with prior findings (18). In contrast, systolic function in banded beclin $1 \mathrm{Tg}$ hearts was greatly diminished (\%FS $=25 \pm 8, n=5)$ as compared with controls $(59 \pm 8, n=5 ; P<0.05$ ) (Figure $10 \mathrm{~F}$ and Table 2). Additional evidence for decompensation was revealed by significant ventricular dilatation in these animals; both LVESD $(P<0.05)$ and LVEDD $(P<0.01)$ were increased in banded Tg mice compared with WT mice (Figure 10, G and H, and Table 2). Together, these data point to stress-induced autophagy, amplified by Beclin 1 overexpression, as promoting deterioration in cardiac performance and pathological increases in heart growth and fibrotic change.

\section{Discussion}

Interest in autophagy has increased in recent years as this highly conserved cellular process has been implicated in a growing number of diseases. Here, we explore the role of cardiomyocyte autophagy in the remodeling response of the LVs subjected to hemodynamic stress. The major findings of this study are as follows: (a) hemodynamic stress is capable of inducing a robust autophagic response in cardiac myocytes; (b) beclin 1 is a required molecular element of the cardiac autophagic response; (c) beclin 1 haploinsufficiency diminishes load-induced autophagy in heart, which is associated with diminished pathological remodeling; and (d) Beclin 1 overexpression amplifies the autophagic response to stress and augments pathological remodeling. Together, these studies implicate cardiac autophagy in the pathogenesis of load-induced heart failure, and they suggest that the machinery of autophagy may be a target for novel therapeutic intervention.

Autophagy in heart disease. Autophagy is a prominent feature of a number of disease processes, yet its role in disease pathogenesis is unclear. With respect to the heart, there are clinical and genetic indications that autophagy is a prominent feature in diseased ventricle. In the 1980s, Wildenthal and coworkers reported a series of studies demonstrating activation of lysosomal pathways of protein degradation in hypoxic or starved ventricle (30). Ventricular biopsies from patients with heart failure show an increase in lysosomal structures, consistent with autophagy $(31,32)$. In certain instances, autophagy has been shown to precede and lead to caspase-independent cell death (32). Recently, cardiac autophagy was reported in models of short-term nutrient deprivation $(15,19,20)$, chronic ischemia (33), diphtheria toxin-induced injury (34), and ischemia-reperfusion injury $(35,36)$.

Other studies from the 1980s based on measurements of autophagosome volume fractions suggested that short-term exposure to isoproterenol (37) or aortic constriction (38) led to decreased autophagy activity in rat heart. At present, an explanation of the divergence of these conclusions from those reported here is not apparent although we believe that present-day molecular approaches may provide more reliable and robust measures of autophagic activity.

A direct genetic link between autophagy and heart disease was suggested by studies of Danon disease, an X-linked cardiomyopa- 


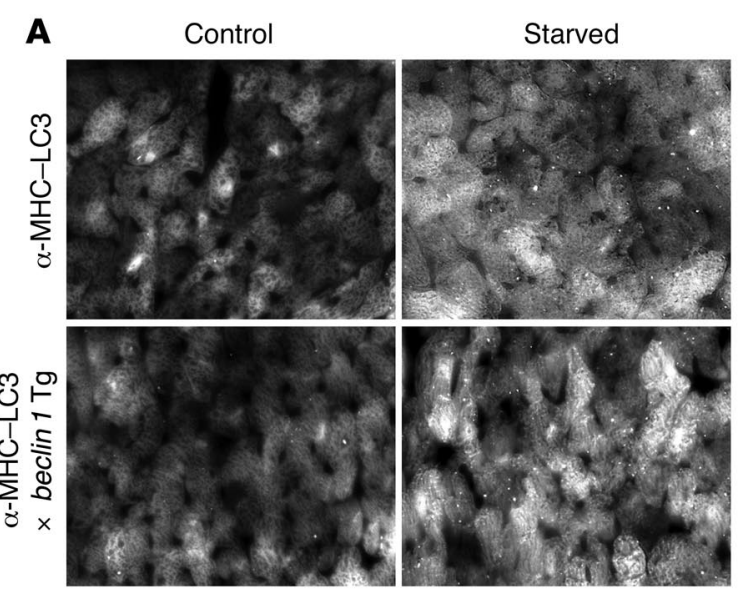

B

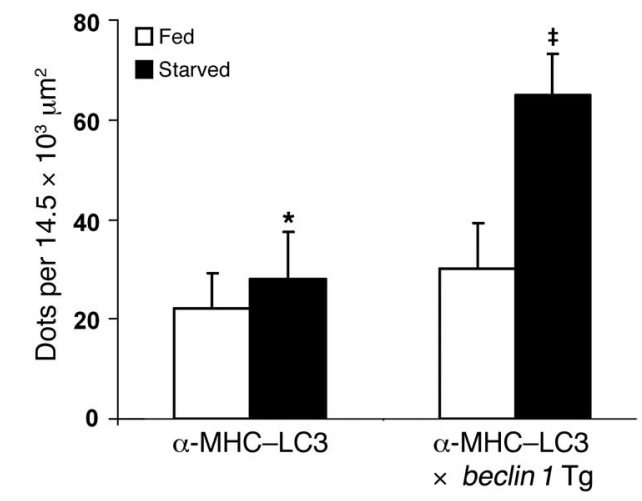

thy that results from deficiency in LAMP-2, a transmembrane protein present in the late endosome and lysosome (39). However, as maturation of autophagosomes is defective in LAMP-2-deficient mice, it is possible that accumulation of autophagosomes in this disorder is a secondary consequence of lysosomal dysfunction and not a primary mechanism of disease. The findings reported here, then, are the first, to our knowledge, to implicate increased autophagic activity in the pathogenesis of heart failure.

Traditionally, detection of autophagy has relied on ultrastructural methods of electron microscopy. This approach, however, hinges critically on careful control of fixation conditions and the user's definition of double-membrane-bound or multilamellar vesicles. When a GFP-tagged LC3 protein is processed, GFP-positive membranes and vesicles form, permitting a quantifiable readout of autophagic activity (15). Here, we have extended this strategy to monitor autophagy specifically in cardiac myocytes using a cardiacspecific $\alpha$-MHC-GFP-LC3 Tg. We confirmed that forced expression of LC3 does not trigger autophagy and that mLC3 migrates to autophagosome membranes and thereby provides a bona fide and quantifiable readout of autophagic activity. Our findings of starvation-induced increases in autophagy, using our cardiomyocytespecific autophagy reporter mouse line, recapitulate results from a GFP-LC3 Tg model driven by the ubiquitously expressed CMV promoter, confirming the validity of our novel reagent (15).

Using our reporter mice, we found that cardiomyocyte autophagy is activated at different stages of load-induced heart failure. In the early phase, autophagic activity was dramatically increased. In the later, sustained phases of the disease, however, autophagy declined to lower levels, though remaining elevated relative to control. In failing heart, autophagic activity remained moderately ele-

\section{Figure 8}

Starvation-induced autophagy is increased in beclin $1 \mathrm{Tg}$ mice. (A) Starvation-induced (24 hours) autophagy, manifested as punctate GFP-LC3 dots, is significantly amplified in beclin $1 \mathrm{Tg}$ hearts. All images were taken from basal septum, and nutrient deprivation was limited to 24 hours, rather than 48 hours, due to the amplified autophagic response in beclin $1 \mathrm{Tg}$ hearts. Scale bar: $35 \mu \mathrm{m}$. (B) Quantification of GFP aggregates per microscopic field $\left(14,479 \mu \mathrm{m}^{2}\right.$, basal septum) demonstrated significant upregulation of autophagic activity in beclin 1 $\mathrm{Tg}$ hearts exposed to starvation. $n=3-5$ microscopic fields in each of 5 mice. ${ }^{\star} P<0.05$ versus fed; $\ddagger P<0.01$ versus fed.

vated. Interestingly, the septum consistently manifested a greater autophagic response than other regions of the LV. The reason for this differential susceptibility to autophagic activation remains obscure at this time. Intriguingly, myocardial scarring in patients with hypertrophic cardiomyopathy is inhomogeneous, and, when present, consistently localizes to the interventricular septum (40).

Autophagy-dependent ventricular remodeling. Declines in ventricular performance are a sensitive marker of pathological remodeling, revealing changes that can precede either reactive hypertrophy or diminished survival. By studying the performance of hearts in which Beclin 1 expression was either up- or downregulated, we were able to detect significant changes in the pathological remodeling of pressure-stressed ventricle. To enhance sensitivity, we employed a model of severe, pressure-overload heart failure, in which LV dysfunction and clinical heart failure develop rapidly (17). Importantly, heterozygous disruption of the gene coding for Beclin 1 does not eliminate autophagic activity completely; this, combined with the fact that mechanisms of ventricular remodeling are multifactorial, likely explains why sTAB-induced declines in ventricular performance were only partially inhibited in beclin $1^{+/}$mice. However, downregulation of autophagy in beclin $1^{+/-}$mice blunted load-induced declines in systolic function by $25 \%(P<0.01)$, which, given the rapidity and severity of the response to $\mathrm{sTAB}$, is likely to be clinically significant. Indeed, we would argue that comparable degrees of preservation of systolic function in patients with heart disease (ejection fraction falling from $65 \%$ to $30 \%$ rather than $20 \%$ ) may well be clinically significant. In support of this notion, data from the Framingham Heart Study demonstrate that modest differences in systolic ventricular function are prognostically significant over the long run (41). In future studies, it will be important to study cardiac autophagy in animal models of chronic disease.

Animals with sTAB-induced heart failure are clinically ill and lethargic, and they suffer increased mortality similar to patients with class IV heart failure. In this model of severe disease, selected to maximize cardiac autophagy, heterozygous inactivation of beclin 1 and consequent diminution in autophagic activity had no effect on survival. It is possible that beclin 1 haploinsufficiency is inadequate to ablate all the potentially detrimental and beneficial effects of cardiac autophagy. As beclin $1^{-1-}$ mice are embryonic lethal, cardiac-specific deletion of beclin 1 or another autophagycritical gene may be necessary to resolve this issue.

At one level, autophagy-mediated protein degradation would be expected to antagonize cell growth. It is paradoxical, then, that augmented autophagy in beclin 1 Tgs was associated with increased cardiac hypertrophy. We speculate that under the conditions studied here, compensatory anabolic mechanisms are activated and culminate in increased heart growth. In the future, it will be important to define those mechanisms. 
A

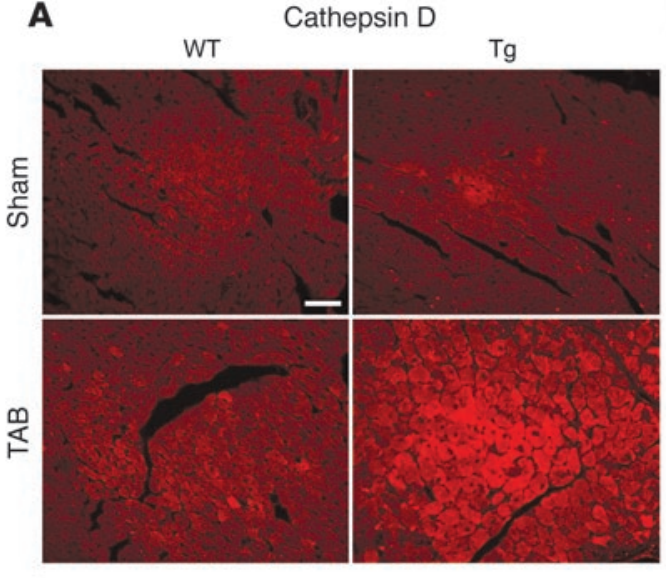

B

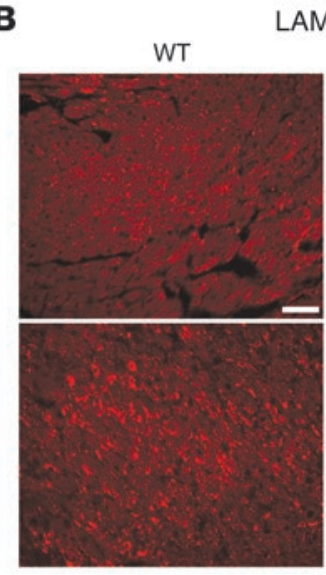

LAMP-1

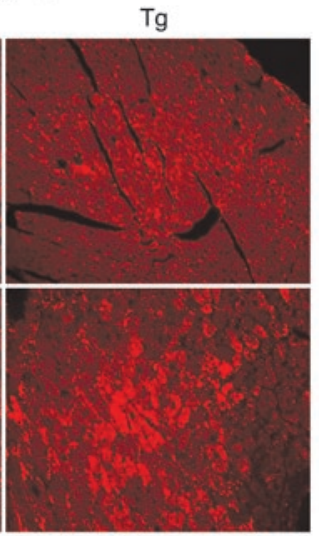

C

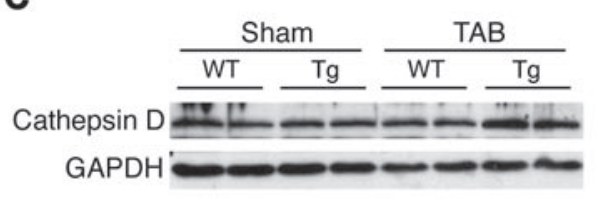

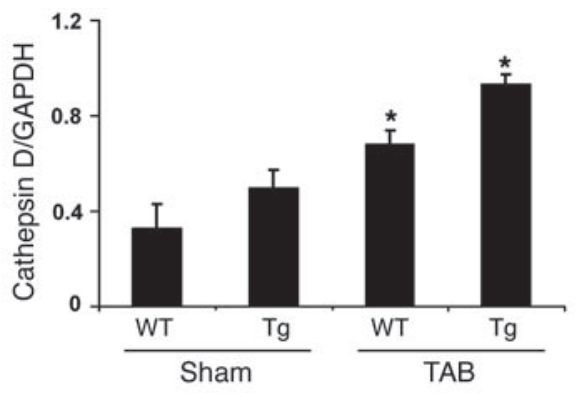

\section{Figure 9}

Increased abundance of lysosomal markers in TAB LVs and in beclin 1 Tgs. Cathepsin D (A) and LAMP-1 (B), detected by immunohistochemistry, are increased in $T A B$ ventricle and in beclin $1 \mathrm{Tg} \mathrm{LV}$, indicative of increased lysosomal activity. Scale bars: $40 \mu \mathrm{m}$. (C) Representative immunoblots probed for cathepsin $D$ in ventricular lysates as indicated. ${ }^{*} P<0.05$ versus WT sham.
Recent studies have uncovered significant interactions between autophagic and apoptotic signaling pathways $(19,22)$. However, we find only very modest increases in apoptosis in both our TAB and sTAB models. Further, both fibrosis and autophagic activity were centered in the basal septum whereas low-level apoptotic activity in cardiomyocytes was randomly distributed throughout the ventricle. All this said, it remains possible that low levels of apoptosis beyond the limits of detection using our methods may contribute to the pathogenesis of disease in our models. However, we have shown here that autophagy changes according to protein Beclin 1 dosage whereas apoptosis does not. This strengthens our argument that the effects of altered Beclin 1 levels are not mediated through apoptosis.

Autophagy in heart failure progression. The precise role of autophagy in heart failure progression remains unclear. Indeed, it is possible that autophagic activity carries out different functions depending on disease stage and severity. In our model of acute-onset hemodynamic stress, amplification of the autophagic response in beclin $1 \mathrm{Tg}$ mice led to increased hypertrophic growth, decreased performance, and increased fibrosis. This suggests that enhanced autophagic activity has pathological consequences, potentially achieved by promoting autophagic cell death. This does not imply, however, that all autophagic activity is necessarily detrimental. On the contrary, we postulate that induction of autophagy in response to stress may be initially beneficial, and there are at least 3 wellestablished mechanisms whereby this might take place.

First, autophagy may be activated to eliminate damaged proteins that accumulate within the cardiomyocyte. Several reports point to functional defects in the ubiquitin-proteasome system (UPS) in heart failure resulting in accumulation of misfolded proteins (42). Consistent with this, we have observed increased ubiquitin staining in pressure-stressed ventricle, which is greatly accentuated in basal septum (data not shown). Further, pathological accumulation of reactive oxygen species plays a role in heart failure (43), which may both damage proteins and inhibit the UPS. Therefore, activation of autophagy at early stages of the disease may be a protective mechanism for scavenging and eliminating misfolded, polyubiquitinated protein aggregates. Work is presently underway to test this hypothesis.

Second, cardiomyocyte autophagy may play a critical role in the removal of damaged organelles, such as mitochondria, to maintain cellular homeostasis and protect against apoptosis. In hepatocytes, opening of the mitochondrial permeability transition pore triggers the autophagic scavenging of individual mitochondria (44). In this context, uncoupling of oxidative phosphorylation renders mitochondria unable to generate ATP and transforms them into futile sinks of ATPase activity. Such mitochondria need to be removed to prevent a catastrophic loss of ATP. This may be one of the critical functions of autophagy in the heart. However, the point at which autophagic activity becomes autophagic death has not been defined. One possibility is that death occurs when too few mitochondria remain after damaged ones have been targeted and cleared.

Third, autophagy may provide a source of energy to compensate for the augmented ATP requirements induced by pressure overload. For many years, it has been inferred that failing heart is relatively energy starved due to a mismatch between ATP supply and demand. Intriguingly, AMP-activated protein kinase, an ATP sensor that is increased in cardiac hypertrophy (45), is linked with autophagy (46). Thus, it is reasonable to hypothesize that cardiomyocyte autophagy recycles cytoplasmic proteins to augment energy supplies and replenish substrate for ATP synthesis.

Perspective. There is a growing need for novel drugs to prevent or reverse pathological cardiac hypertrophy and heart failure. Efforts 
Table 2

Echocardiographic, morphometric, and physiological parameters 3 weeks post TAB surgery

\begin{tabular}{|c|c|c|c|c|}
\hline & \multicolumn{2}{|c|}{ Sham } & \multicolumn{2}{|c|}{ TAB } \\
\hline & WT & Beclin $1 \mathrm{Tg}$ & WT & Beclin $1 \mathrm{Tg}$ \\
\hline \multicolumn{5}{|c|}{ Echocardiographic data } \\
\hline $\mathrm{HR}(\mathrm{bpm})$ & $660 \pm 67$ & $646 \pm 65$ & $624 \pm 68$ & $564 \pm 80$ \\
\hline IVSD (mm) & $0.95 \pm 0.09$ & $0.89 \pm 0.08$ & $0.99 \pm 0.17$ & $1.13 \pm 0.25$ \\
\hline LVPW (mm) & $1.13 \pm 0.15$ & $1.22 \pm 0.10$ & $1.17 \pm 0.12$ & $1.38 \pm 0.25$ \\
\hline /EDD (mm) & $3.51 \pm 0.51$ & $3.57 \pm 0.50$ & $3.32 \pm 0.23$ & $4.25 \pm 0.21^{B}$ \\
\hline LVESD (mm) & $1.35 \pm 0.38$ & $1.33 \pm 0.43$ & $1.35 \pm 0.23$ & $3.19 \pm 0.38^{B}$ \\
\hline$\% \mathrm{FS}$ & $62.2 \pm 5.84$ & $63.23 \pm 8.45$ & $59.16 \pm 7.69$ & $24.88 \pm 7.90^{B}$ \\
\hline$n$ (animals) & 6 & 6 & 5 & 5 \\
\hline \multicolumn{5}{|c|}{ Morphometric and physiological data } \\
\hline BW (g) & $25.1 \pm 0.6$ & $25.2 \pm 1.3$ & $24.2 \pm 0.6$ & $24.1 \pm 0.9$ \\
\hline W/BW & $5.10 \pm 0.47$ & $5.40 \pm 0.5$ & $6.28 \pm 0.78^{A}$ & $8.86 \pm 1.75^{B}$ \\
\hline LW (r & $160 \pm 10.5$ & $151.1 \pm 12.0$ & $146.4 \pm 9.5$ & $384.0 \pm 55.7^{A}$ \\
\hline $\mathrm{SBP}(\mathrm{mmHg})$ & $133.3 \pm 10$ & $132.4 \pm 9.7$ & $119 \pm 7.1^{\mathrm{A}}$ & $108.6 \pm 10.2^{B}$ \\
\hline$n$ (animals) & 6 & 6 & 8 & 6 \\
\hline \multicolumn{5}{|c|}{ Invasive hemodynamics } \\
\hline HR (bpm) & $480 \pm 16$ & $455 \pm 21$ & $409 \pm 53$ & $459 \pm 7$ \\
\hline$d P / d t_{\max }(\mathrm{mmHg} / \mathrm{s})$ & $5,757 \pm 545$ & $5,969 \pm 228$ & $4,344 \pm 248$ & $3,181 \pm 350^{\mathrm{B}}$ \\
\hline$d P / d t_{\min }(\mathrm{mmHg} / \mathrm{s})$ & $-5,624 \pm 503$ & $-5,235 \pm 663$ & $-4,260 \pm 427$ & $-2,967 \pm 614^{B}$ \\
\hline $\operatorname{LVSP}(\mathrm{mmHg})$ & $92.8 \pm 8.6$ & $81.8 \pm 13$ & $94.6 \pm 9.5$ & $75.4 \pm 14.6$ \\
\hline$n$ (animals) & 7 & 5 & 5 & 5 \\
\hline
\end{tabular}

${ }^{A} P<0.05$ versus WT sham; ${ }^{B} P<0.05$ versus WT TAB.

to target autophagy selectively in the heart may be a rational step toward the development of novel strategies to prevent heart failure in humans. Furthermore, there are drugs already in clinical use that have been demonstrated to have an impact on the process of autophagy. Finally, because autophagic activity is associated with a number of skeletal muscle and neurological disorders, findings reported here may have relevance to these diseases.

\section{Methods}

Preparation of LC3 antibody. LC3 anti-peptide antibody was produced by chemically coupling murine LC3 (amino acids $2-15$ plus a C terminal cysteine, PSEKTFKQRRSFEQC) to keyhole limpet hemocyanin. New Zealand white rabbits were immunized subcutaneously, and the sera were affinity purified. Discrete bands at $18 \mathrm{kDa}$ and $16 \mathrm{kDa}$ were detected whose relative abundance shifted dramatically with short-term starvation (an established trigger of autophagy), consistent with LC3-I and LC3-II, respectively (Supplemental Figures 1 and 2).

Expression constructs. mLC3 cDNA was PCR amplified from a cDNA library (5'-GGCCGGAGATCTATGCCGTCCGAGAAGACCTTCAAG-3', forward primer; 5'-GGCCGGGAATTCCTTTGTTGGAGTCTTACACAG-3', reverse primer) and subcloned into the $C$ terminus of enhanced GFP (EGFP-C1) plasmid (BD Biosciences - Clontech; BglII and EcoRI sites). Myc-LC3 was engineered by inserting PCR-amplified mLC3 into pcDNA3$\mathrm{N}$-myc plasmid. rLC3 cDNA was amplified from rat brain by RT-PCR (5'-CGCGCCATGCCGTCCGAGAAGAC-3', forward primer; 5'-GTCTGTCACAAGCATGGCTCTCT-3', reverse primer) and inserted into the TA cloning vector pCR2.1 (Invitrogen). For EGFP-rLC3, the rLC3 open reading frame from TA-LC3 vector was introduced into EGFP-C1 plasmid.

Cell culture and transfection. C2C12 skeletal myoblasts were maintained in DMEM supplemented with $20 \%$ FCS. One day before transfection, cells were seeded onto a $35-\mathrm{mm}$ dish $\left(1.5 \times 10^{5}\right.$ per well). Plasmids were transfected using Lipofectamine 2000 (Invitrogen) according to the manufac- turer's protocol. Twenty-four hours after transfection, cells were subjected to amino acid starvation (2 hours) and then harvested.

Cardiomyocyte autophagy reporter Tgs. A 1.2-kb fragment containing an EGFP-mLC3 fusion gene was cloned into an $\alpha$-MHC promoter construct (SalI and HindIII sites). A NotI fragment harboring the $\alpha$-MHC promoter (5.5 kb), EGFP-LC3 fusion gene, and human growth hormone polyA signal $(0.6 \mathrm{~kb})$ was excised and purified by GELase according to the manufacturer's protocol (EPICENTRE Biotechnologies). The DNA fragment was further purified using Elutip-d columns (Schleicher \& Schuell) and microinjected into fertilized oocytes from C57BL/6 mice. Tg founders were identified by PCR (5'CATCGAGCTGAAGGGCATCG-3', forward primer; 5' CTATAATCACTGGGATCTTGGTG-3', reverse primer). Eleven founder mice were generated, each of which was healthy and fertile. Tg lines were maintained by crossing with C57BL/6. No significant abnormalities of heart development, heart size, or function were observed in the Tg mice as compared with the WT control.

Beclin 1 targeted mutant mice and Beclin 1 Tgs. Mouse beclin 1 cDNA with a carboxyterminal HA epitope tag was cloned into an $\alpha$-MHC promoter construct (SalI and HindIII sites). A fragment containing the $\alpha$-MHC promoter, HA-tagged beclin 1, and human growth hormone polyA was released by BamHI and purified for microinjection. Three founder mice were generated, each of which was healthy and fertile. Tg lines were maintained by crossing with C57BL/6, and offspring from 2 lines $(17,18)$ were used for further study. No significant abnormalities of heart development, heart size, or function were observed in the Tg mice as compared with the WT controls.

Beclin $1^{+/-}$mice were developed as $129 S v / J \times C 57 B L / 6$ crosses and backcrossed to C57BL/ 6 mice for 20 or more generations for experimental studies (12). Beclin $1^{+/-}$129Sv/J.C57BL/6 or $\alpha-M H C-$ beclin 1 C57BL/ 6 crossed with $\alpha-M H C-L C 3 C 57 B L / 6$ Tgs were used to analyze autophagy in vivo, and $\alpha-M H C-L C 3$ Tgs were used as control. Banded $\alpha-M H C-$ beclin 1 C57BL/6 or beclin $1^{+/-} 129 \mathrm{~Sv} / \mathrm{J}$.C57BL/ 6 crosses were used for studies of pressure overload cardiac hypertrophy, and WT littermates were used as controls. All experiments were conducted with 6- to 8-week-old male mice and were approved by the Institutional Animal Care and Use Committee of The University of Texas Southwestern Medical Center at Dallas.

$T A B$. Mice (6- to 8-week-old males) were subjected to pressure overload by $\mathrm{TAB}(47)$ or $\mathrm{STAB}(17)$. TAB induces stable, compensated ventricular hypertrophy with preserved ventricular function (18), and sTAB induces pressure-overload heart failure (17). At 3 weeks, when the hypertrophic response reached steady-state, integrity of aortic banding was confirmed by inspection of the surgical constriction and by visualization of marked differences in caliber of the right and left carotid arteries. In some animals, transstenotic pressures were measured as described (18). Sham-operated animals underwent an identical operation without imposition of aortic stenosis.

Tail cuff blood pressure recordings. Systolic blood pressure was recorded in mice using a noninvasive blood pressure tail cuff system (ADInstruments). Before testing, all mice were trained to become accustomed to handling and short-term restraint. Baseline blood pressure was recorded for at least 4 consecutive days at $32^{\circ} \mathrm{C}$ (Deltaphase isothermal pad; Braintree Scientific Inc.), and at least 10 readings were made per mouse at each session. We were unable to record systolic blood pressures reliably in our pressure-overload (sTAB) mice owing to increased periprocedural mortality and dampened waveforms in these severely ill mice with systolic dysfunction. 


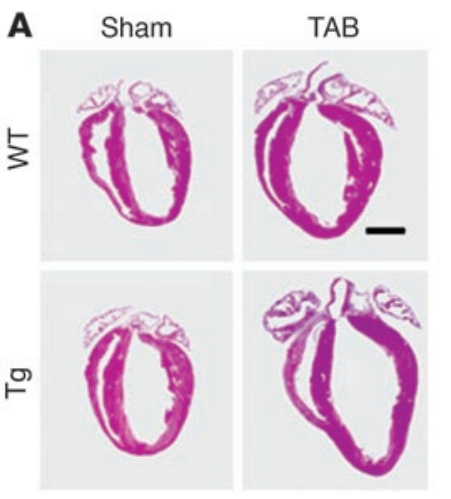

B
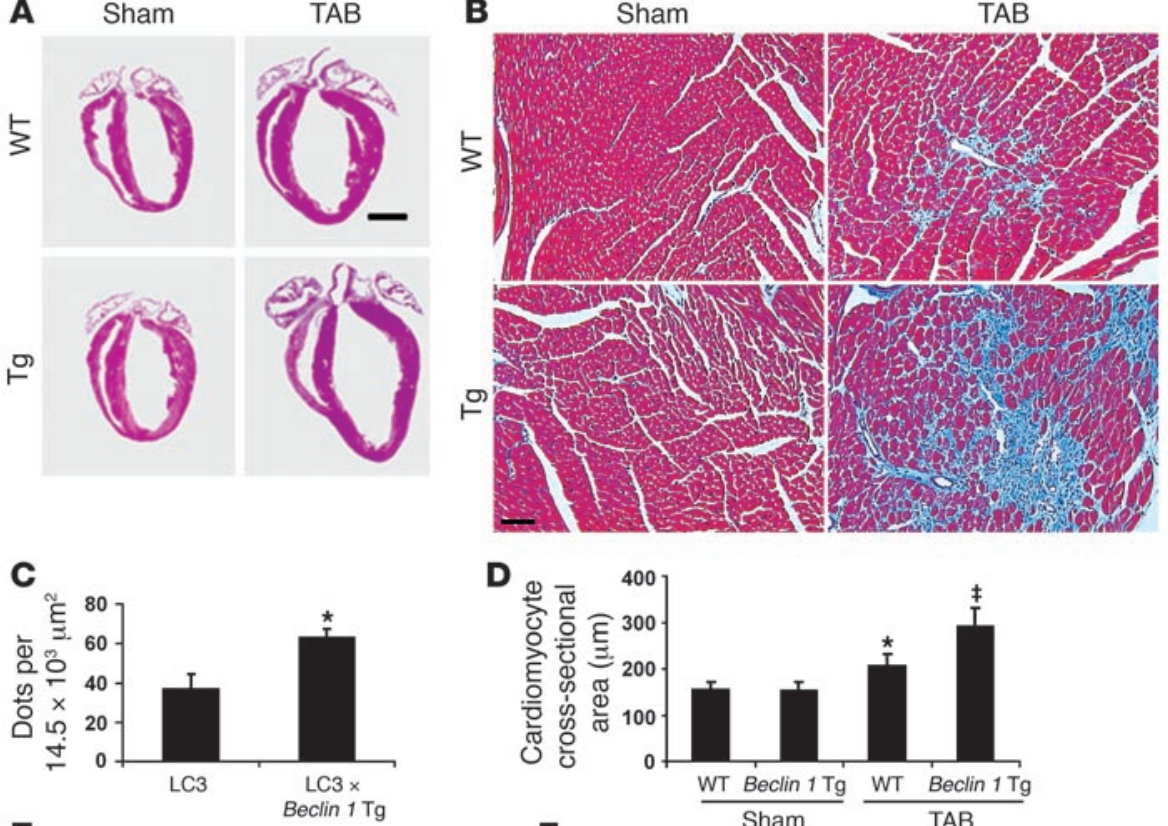

E

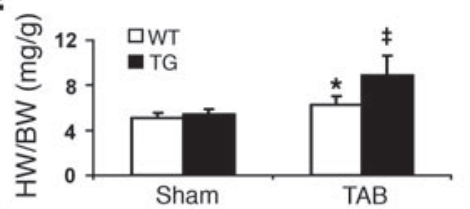

G

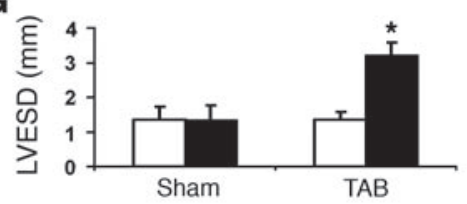

D
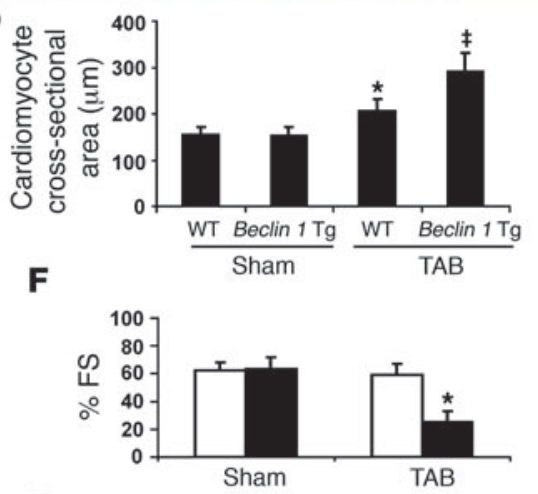

H

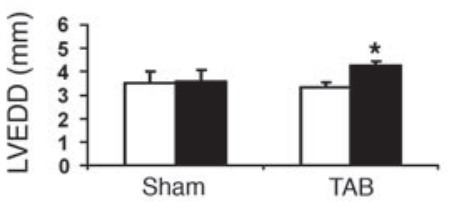

\section{Figure 10}

Stress-induced autophagy is increased in beclin $1 \mathrm{Tg}$ mice. (A) Four-chamber sections of hearts treated as listed and harvested at 3 weeks. Scale bar: $2 \mathrm{~mm}$. (B) Trichrome staining for collagen (blue) demonstrates increased fibrotic change in banded beclin 1 Tg hearts. Scale bar: $60 \mu \mathrm{m}$. (C) Quantification of GFP aggregates per microscopic field $\left(14,479 \mu \mathrm{m}^{2}\right.$, basal septum) demonstrates significant upregulation of autophagic activity in beclin $1 \mathrm{Tg}$ hearts exposed to standard TAB. $n=3-5$ microscopic fields in each of 3 mice. ${ }^{*} P<0.05$ versus sham. (D) Twodimensional cardiomyocyte cross-sectional area measured as described. $\ddagger P<0.05$ versus WT TAB. (E) Banding-induced heart growth, measured as HW/BW, is amplified in beclin $1 \mathrm{Tg}$ mice compared with WT controls. $n=6 \mathrm{WT}$ sham; $n=6 \mathrm{Tg}$ sham; $n=8 \mathrm{WT}$ TAB; $n=6$ Tg TAB. (F) In WT mice, moderate pressure stress induced by $T A B$ did not alter systolic function. In beclin $1 \mathrm{Tg}$ mice, however, \%FS was diminished significantly at 3 weeks. Ventricular decompensation was observed in beclin $1 \mathrm{Tg}$ mice exposed to TAB, as LVESD $(\mathbf{G})$ and LVEDD $(\mathbf{H})$ were both increased significantly at 3 weeks compared with control. $n=6$ WT sham; $n=6 \mathrm{Tg}$ sham; $n=5$ WT TAB; $n=5 \mathrm{Tg}$ TAB.
Western blotting. Proteins were fractionated by SDS-PAGE and transferred onto a nitrocellulose membrane. The blot was blocked (1 hour, room temperature $[\mathrm{RT}]$ ) with Tris-buffered saline (TBS) containing 0.1\% Tween-20 and $5 \%$ nonfat milk and then probed with primary antibody. The blot was washed $(4 \times 10 \mathrm{~min})$ with TBS containing $0.1 \%$ Tween- 20 , followed by incubation with HRP-conjugated secondary antibody (1:5,000, 1 hour, $\mathrm{RT})$. Blots were washed $(4 \times 10 \mathrm{~min})$ with TBS containing $0.1 \%$ Tween-20 and revealed by ECL Plus (Amersham Biosciences).

Histology and immunocytochemistry. Hearts were perfusion fixed with ice-cold heparinized saline and 4\% paraformaldehyde in PBS followed by immersion fixation ( 4 hours at $4^{\circ} \mathrm{C}$ with agitation). Hearts for GFP analysis were cryoprotected in $30 \%$ sucrose overnight. After embedding in freezing matrix (TFM, Triangle Bioscience), cryostat sections were prepared ( $8 \mu \mathrm{m}$, Leica CM3000 cryostat), air-dried, and stored at $-80^{\circ} \mathrm{C}$ until coverslipping and photography. Hearts for routine histology, Masson trichrome, LAMP-1 and cathepsin D immunostaining, and TUNEL reactions were rinsed, dehydrated, cleared, and embedded in frontal orientation in paraffin. Paraffin sections were prepared $(5 \mu \mathrm{m}$, Leica RM2255 rotary microtome) and stored at RT until staining. LAMP-1 and cathepsin $\mathrm{D}$ immunostaining on paraffin sections was performed with minimal antigen retrieval $(0.3 \%$ Triton X-100) using commercially available antisera (rat anti-LAMP-1, 1:500; Santa Cruz Biotechnology Inc.; rabbit anti-cathepsin D, 1:100; Santa Cruz Biotechnology Inc.). TUNEL staining was conducted using a commercially available kit
(DeadEnd Fluorometric TUNEL System; Promega). C2C12 myoblasts on coverslips were rinsed with PBS+ (PBS containing $\mathrm{Ca}^{2+}$ and $\mathrm{Mg}^{2+}$ ), briefly fixed with ice-cold $4 \%$ paraformaldehyde in PBS+, and processed for immunostaining, as above.

Electron microscopy. Hearts were retrograde perfused using PBS and $2 \%$ glutaraldehyde in $0.1 \mathrm{M}$ cacodylate buffer. Postfixation occurred in $2 \%$ osmium tetroxide in $0.1 \mathrm{M}$ cacodylate buffer and $1 \%$ aqueous uranyl acetate, each for 1 hour. An ascending series of ethanol washes $(50 \%, 70 \%$, $90 \%, 100 \%$ ) was used followed by transitioning to propylene oxide and then a 1:1 mixture of propylene oxide and EMbed 812 (Electron Microscopy Sciences). The tissue was incubated in EMbed for 1 hour, then placed in a $70^{\circ} \mathrm{C}$ oven to polymerize. Sections $(75-80 \mathrm{~nm})$ were cut using a Leica Ultramicrotome and a Diatome diamond knife, collected on 200-mesh copper grids and poststained in 5\% uranyl acetate in ethanol (10 minutes) and Reynold's lead citrate (5 minutes). A JEOL 1200 EX transmission electron microscope, operating at $40-120 \mathrm{kV}$ and equipped with a digital camera, was used to image the sections.

RNA dot blots. Total RNA was isolated from the LVs using the RNeasy Midi Kit (QIAGEN) according to the manufacturer's recommended protocol. RNA dot blot analysis was performed as previously described (48).

Cardiomyocyte cross-sectional area. Cardiomyocyte cross-sectional areas were measured as described previously (49).

Echocardiography. Transthoracic echocardiograms were recorded in conscious, sedated mice as described $(18,48)$. In brief, views were taken 
in planes which approximated the parasternal short-axis view (chordal level) and the apical long-axis view in humans. LV internal diameters and wall thicknesses were measured (at least 3 cardiac cycles) at end-systole and end-diastole. LV mass and volumes were calculated using the arealength method (50).

Reagents. Reagents were procured as follows: Cy3-conjugated goat antirabbit and Cy3-conjugated donkey anti-rat (Jackson ImmunoResearch Laboratories Inc.), goat anti-rabbit (Sigma-Aldrich), Lipofectamine 2000 (Invitrogen), OptiMEM (Invitrogen), Elutip-d columns (Schleicher \& Schuell), GELase (EPICENTRE Technologies), rabbit anti-c-Myc (SigmaAldrich), rabbit anti-GFP (Invitrogen), $\alpha$-tubulin monoclonal (SigmaAldrich), and rabbit anti-GAPDH (Santa Cruz Biotechnology Inc.).

Statistics. Averaged data are reported as mean \pm SD. Statistical significance was analyzed using Student's unpaired $t$ test or 1-way ANOVA followed by Bonferroni's method for post hoc pairwise multiple comparisons.

\section{Acknowledgments}

We gratefully acknowledge Chris Gilpin for assistance with electron microscopy. This work was supported by grants from the Donald W. Reynolds Cardiovascular Clinical Research Center (to J.A. Hill), the NIH (HL-075173, HL-080144, and HL-006296 to J.A. Hill; HL-072016 to B.A. Rothermel), and the American Heart Association (0640084N to J.A. Hill; 0655202Y to B.A. Rothermel).

Received for publication November 29, 2005, and accepted in revised form May 8, 2007.

Address correspondence to: Joseph A. Hill, Division of Cardiology, UT Southwestern Medical Center, NB11.200, 6000 Harry Hines Boulevard, Dallas, Texas 75390-8573, USA. Phone: (214) 648-1400; Fax: (214) 648-1450; E-mail: joseph.hill@utsouthwestern.edu.
1. Rosamond, W., et al. 2007. Heart disease and stroke statistics - 2007 update. A report from the American Heart Association Statistics Committee and Stroke Statistics Subcommittee. Circulation. 115:e69-e171. doi:10.1161/circulationaha.105.171600.

2. Frey, N., Katus, H.A., Olson, E.N., and Hill, J.A. 2004. Hypertrophy of the heart: a new therapeutic target? Circulation. 109:1580-1589.

3. Heineke, J., and Molkentin, J.D. 2006. Regulation of cardiac hypertrophy by intracellular signalling pathways. Nat. Rev. Mol. Cell Biol. 7:589-600.

4. Klionsky, D.J., and Emr, S.D. 2000. Autophagy as a regulated pathway of cellular degradation. Science. 290:1717-1721.

5. Levine, B., and Klionsky, D.J. 2004. Development by self-digestion: molecular mechanisms and biological functions of autophagy. Dev. Cell. 6:463-477.

6. Mizushima, N. 2005. The pleiotropic role of autophagy: from protein metabolism to bactericide. Cell Death Differ. 12(Suppl. 2):1535-1541.

7. Lum, J.J., DeBerardinis, R.J., and Thompson, C.B. 2005. Autophagy in metazoans: cell survival in the land of plenty. Nat. Rev. Mol. Cell Biol. 6:439-448.

8. Larsen, K.E., and Sulzer, D. 2002. Autophagy in neurons: a review. Histol. Histopathol. 17:897-908.

9. Hara, T., et al. 2006. Suppression of basal autophagy in neural cells causes neurodegenerative disease in mice. Nature. 441:885-889.

10. Komatsu, M., et al. 2006. Loss of autophagy in the central nervous system causes neurodegeneration in mice. Nature. 441:880-884.

11. Yue, Z., Jin, S., Yang, C., Levine, A.J., and Heintz, N. 2003. Beclin 1, an autophagy gene essential for early embryonic development, is a haploinsufficient tumor suppressor. Proc. Natl. Acad. Sci. U. S. A. 100:15077-15082.

12. Qu, X., et al. 2003. Promotion of tumorigenesis by heterozygous disruption of the beclin 1 autophagy gene. J. Clin. Invest. 112:1809-1820. doi:10.1172/ JCI200320039.

13. Gutierrez, M.G., et al. 2004. Autophagy is a defense mechanism inhibiting BCG and Mycobacterium tuberculosis survival in infected macrophages. Cell. 119:753-766.

14. Kabeya, Y., et al. 2000. LC3, a mammalian homologue of yeast Apg8p, is localized in autophagosome membranes after processing. EMBOJ. 19:5720-5728.

15. Mizushima, N., Yamamoto, A., Matsui, M., Yoshimori, T., and Ohsumi, Y. 2004. In vivo analysis of autophagy in response to nutrient starvation using transgenic mice expressing a fluorescent autophagosome marker. Mol. Biol. Cell. 15:1101-1111.

16. Berenji, K., Drazner, M.H., Rothermel, B.A., and Hill, J.A. 2005. Does load-induced ventricular hypertrophy progress to systolic heart failure? Am. J. Physiol. Heart Circ. Physiol. 289:H8-H16.

17. Rothermel, B.A., et al. 2005. Differential activation of stress-response signaling in load-induced cardiac hypertrophy and failure. Physiol. Genomics. 23:18-27.

18. Hill, J.A., et al. 2000. Cardiac hypertrophy is not a required compensatory response to short-term pressure overload. Circulation. 101:2863-2869.

19. Pattingre, S., et al. 2005. Bcl-2 antiapoptotic proteins inhibit beclin-1-dependent autophagy. Cell. 122:927-933.

20. Kuma, A., et al. 2004. The role of autophagy during the early neonatal starvation period. Nature. 432:1032-1036.

21. Kihara, A., Noda, T., Ishihara, N., and Ohsumi, Y. 2001. Two distinct Vps34 phosphatidylinositol 3-kinase complexes function in autophagy and carboxypeptidase Y sorting in Saccharomyces cerevisiae. J. Cell Biol. 152:519-530.

22. Yousefi, S., et al. 2006. Calpain-mediated cleavage of Atg5 switches autophagy to apoptosis. Nat. Cell Biol. 8:1124-1132.

23. Kang, P.M., and Izumo, S. 2000. Apoptosis and heart failure - a critical review of the literature. Circ. Res. 86:1107-1113.

24. Narula, J., Kolodgie, F.D., and Virmani, R. 2000. Apoptosis and cardiomyopathy. Curr. Opin. Cardiol. 15:183-188.

25. Gonzalez, A., et al. 2003. Cardiomyocyte apoptosis in hypertensive cardiomyopathy. Cardiovasc. Res. 59:549-562.

26. Wencker, D., et al. 2003. A mechanistic role for cardiac myocyte apoptosis in heart failure. J. Clin. Invest. 111:1497-1504. doi:10.1172/JCI200317664.

27. Shintani, T., and Klionsky, D.J. 2004. Autophagy in health and disease: a double-edged sword. Science. 306:990-995.

28. Yu, L., et al. 2006. Autophagic programmed cell death by selective catalase degradation. Proc. Natl. Acad. Sci. U. S. A. 103:4952-4957.

29. Liang, X.H., et al. 1999. Induction of autophagy and inhibition of tumorigenesis by beclin 1. Nature. 402:672-676.

30. Decker, R.S., Decker, M.L., Herring, G.H., Morton, P.C., and Wildenthal, K. 1980. Lysosomal vacuolar apparatus of cardiac myocytes in heart of starved and refed rabbits. J. Mol. Cell. Cardiol. 12:1175-1189.

31. Yamamoto, S., Sawada, K., Shimomura, H., Kawamura, K., and James, T.N. 2000. On the nature of cell death during remodeling of hypertrophied human myocardium. J. Mol. Cell. Cardiol. 32:161-175.

32. Lockshin, R.A., and Zakeri, Z. 2002. Caspase-independent cell deaths. Curr. Opin. Cell Biol. 14:727-733.

33. Yan, L., et al. 2005. Autophagy in chronically ischemic myocardium. Proc. Natl. Acad. Sci. U. S. A. 102:13807-13812.

34. Akazawa, H., et al. 2004. Diphtheria toxin-induced autophagic cardiomyocyte death plays a pathogenic role in mouse model of heart failure. J. Biol. Chem. 279:41095-41103.

35. Hamacher-Brady, A., Brady, N.R., and Gottlieb, R.A. 2006. Enhancing macroautophagy protects against ischemia/reperfusion injury in cardiac myocytes. J. Biol. Chem. 281:29776-29787.

36. Matsui, Y., et al. 2007. Distinct roles of autophagy in the heart during ischemia and reperfusion: roles of AMP-activated protein kinase and Beclin 1 in mediating autophagy. Circ. Res. 100:914-922.

37. Pfeifer, U., Fohr, J., Wilhelm, W., and Dammrich, J. 1987. Short-term inhibition of cardiac cellular autophagy by isoproterenol. J. Mol. Cell. Cardiol. 19:1179-1184.

38. Pfeifer, U., and Dammrich, J. 1986. Intracellular turnover and cardiac hypertrophy. Basic Res. Cardiol. 81(Suppl. 1):139-146

39. Tanaka, Y., et al. 2000. Accumulation of autophagic vacuoles and cardiomyopathy in LAMP-2-deficient mice. Nature. 406:902-906.

40. Choudhury, L., et al. 2002. Myocardial scarring in asymptomatic or mildly symptomatic patients with hypertrophic cardiomyopathy. J. Am. Coll. Cardiol. 40:2156-2164.

41. Wang, T.J., et al. 2003. Natural history of asymptomatic left ventricular systolic dysfunction in the community. Circulation. 108:977-982.

42. Dong, X., et al. 2004. In situ dynamically monitoring the proteolytic function of the ubiquitin-proteasome system in cultured cardiac myocytes. Am. J. Physiol. Heart Circ. Physiol. 287:H1417-H1425.

43. Sawyer, D.B., et al. 2002. Role of oxidative stress in myocardial hypertrophy and failure. J. Mol. Cell. Cardiol. 34:379-388.

44. Rodriguez-Enriquez, S., He, L., and Lemasters, J.J. 2004. Role of mitochondrial permeability transition pores in mitochondrial autophagy. Int. J. Biochem. Cell Biol. 36:2463-2472.

45. Tian, R., Musi, N., D’Agostino, J., Hirshman, M.F., and Goodyear, L.J. 2001. Increased adenosine monophosphate-activated protein kinase activity in rat hearts with pressure-overload hypertrophy. Circulation. 104:1664-1669.

46. Liang, J., et al. 2007. The energy sensing LKB1AMPK pathway regulates $\mathrm{p} 27$ (kip1) phosphorylation mediating the decision to enter autophagy or apoptosis. Nat. Cell Biol. 9:218-224.

47. Rockman, H.A., et al. 1991. Segregation of atrialspecific and inducible expression of an atrial natriuretic factor transgene in an in vivo murine model of cardiac hypertrophy. Proc. Natl. Acad. Sci. U. S. A. 88:8277-8281.

48. Hill, J.A., et al. 2002. Targeted inhibition of calcineurin in pressure-overload cardiac hypertrophy. Preservation of systolic function. J. Biol. Chem. 277:10251-10255.

49. Kong, Y., et al. 2006. Suppression of class I and II histone deacetylases blunts pressure-overload cardiac hypertrophy. Circulation. 113:2579-2588.

50. Schiller, N.B., et al. 1979. Left ventricular volume from paired biplane two-dimensional echocardiography. Circulation. 60:547-555. 